\title{
Computer programs to facilitate detailed analysis of how people study text passages
}

\author{
KEITH A. WOLLEN, ROBERT S. CONE, MATTHEW G. MARGRES, and BRUCE P. WOLLEN \\ Washington State University, Pullman, Washington
}

\begin{abstract}
Three computer programs are described for an IBM Personal Computer; these programs enable the gathering of detailed data on reading and study processes. The programs (A) present any text of the experimenter's choosing and permit subjects to study the lines and pages of that text in any order; (B) allow for the subject's use of a light pen to record study time per line, to answer questions, and to indicate lines that are, for instance, important or difficult by "highlighting" them (i.e, changing them to reverse video); (C) allow for multiple study sessions and a review session; (D) present and score multiple-choice questions; (E) permit the use of fill-in test items; (F) calculate 176 values, including detailed data on highlighting, quality of study, testing, and times and frequencies on 18 different categories of lines in study and in review; and (G) make a printout of the raw data and the 176 summary statistics. Also described is one example of a 28-page text and a test that have been used in conjunction with the programs. Data are provided to illustrate the usefulness of the programs.
\end{abstract}

People have been interested for centuries in how people learn from text, but only recently has rigorous research been conducted. Most available research has to do with the study of brief passages quite unlike those encountered in real life (e.g., Brown \& Smiley, 1978; Pichert \& Anderson, 1977; Sulin \& Dooling, 1974; Thorndyke, 1977). Studies of longer passages have generally been limited to rather gross measures, such as time spent studying a passage and the total number of correct answers on a test (e.g., Annis \& Davis, 1978; Crouse \& Idstein, 1972; Fass \& Schumacher, 1978).

A major reason for the lack of detailed research is that detailed study of longer passages has become feasible only with the advent of inexpensive computers. This paper describes computer programs that present lengthy passages to subjects, present and score questions, record time and frequency data, and calculate many different results.

The most obvious goal when studying text is to learn the important information. As Anderson and Pearson (1984) point out, "Perhaps the most pervasive and consistent finding of research on discourse is that important text elements are more likely to be learned and remembered than less important elements"' (p. 272). A key question is why subjects emphasize important material. One likely answer is provided by the attention hypothesis (e.g., Anderson \& Pearson, 1984; Anderson \& Armbruster, 1984). According to this view, subjects attend more to important elements, thereby learning and retaining them

\footnotetext{
Appreciation is expressed to John Hinson, Ronald Hopkins, and John Wright for their comments on this manuscript. Appreciation is also expressed to Craig Wollen for the preparation of Figure 1, and to Ed Miller and Laura Wilson for their contributions to the analysis. Requests for reprints should be sent to Keith A. Wollen, Department of Psychology, Washington State University, Pullman, WA 99164.
}

better. The importance of time has also been emphasized by Faw and Waller (1976) who state that a "major difficulty with much of the available research is that inadequate attention has been paid to the study time variable"' (p. 703).

The prevailing view has been that increased attention to a text segment will be reflected by an increase in the time spent studying that segment. The approach described in this paper enables an assessment of the selective attention theory by measuring the length of time a subject studies each line of text. By using an individual line of text as the unit of analysis, it is possible to obtain a more detailed analysis than is possible with the typical procedure of timing how long subjects spend on larger blocks of text (e.g., Reynolds, Standiford, \& Anderson, 1979) or on entire passages (e.g., Annis \& Davis, 1978).

Another possibility is that selective attention can occur without requiring longer time (e.g., Britton, Meyer, Simpson, Holdredge, and Curry, 1979). For example, a subject can spend about the same study time on each line of text, but daydream on some lines and concentrate on others. The programs presented in this paper permit an assessment of this type of selective attention if subjects are asked to select (by highlighting) the lines they attend to the most.

The highlighting feature is also of potential benefit for those who wish to study metacognition. A common approach in such experiments is to ask subjects, at the end of study, how well they understand the text, how difficult the text is, and how well they think they will do on a test (e.g., Raphael, Myers, Tirre, Fritz, \& Freebody ${ }_{2}$ 1981). With the approach described here, subjects can be asked to highlight lines they understand (or do not understand), or to highlight lines they feel are particularly difficult. Also, subjects can be asked, for instance, to highlight a 
blank line inserted at the bottom of a page if they feel confident they would do well on a question regarding material covered on that page. By such techniques, researchers can obtain evidence concerning metacognition during rather than after study.

Another dependent variable that can be examined with the programs is "lookbacks," which involve a subject's reexamining portions of text previously covered. Lookbacks occur during initial study and are to be distinguished from review that occurs only after initial study is complete, and usually after an interval of time.

Most experimenters view lookbacks as activities that occur when subjects have comprehension and/or memory failures (e.g., Alessi, Anderson, \& Goetz, 1979; Garner, Macready, \& Wagoner, 1984; Garner \& Reis, 1981). Looking back to relevant lines can resolve such failures and provide significant information about metacognition. However, an alternative view is that lookbacks represent a rehearsal activity that avoids comprehension or memory failures in the first place. Our programs offer an objective and easy method for determining the number of lookbacks for each line of text. One need not build ambiguities into the text or use elaborate procedures to document that a lookback has occurred, as some researchers have done (e.g., Garner \& Reis, 1981).

The procedures described here have the additional advantage of permitting research on the review process and its relationship to original study. Like lookbacks, the lines reviewed can provide a great deal of information on what the subject considers important and/or does not understand or remember. Review is obviously an integral part of real-world study techniques, but it has received little research attention.

Although a computer holds great promise for research in reading and studying, there have been relatively few attempts to use computers for the presentation of text and the collection of data. One exception is the research by Reynolds et al. (1979), who presented and recorded time on 4-line text segments. However, time was not obtained for individual lines, the segments frequently consisted of sentence fragments, and subjects were not free to return to previous text segments. Thus, if the beginning of a segment were the end of a confusing sentence, then subjects could not look back at the beginning of the sentence to resolve their confusions.

Another approach (Wollen, Cone, Britcher, \& Mindemann, 1985) involves mounting pages of text on a monitor table equipped with a sliding cursor that indicates the line of text the subject is reading. This method provides a line-by-line analysis of study time, but it is difficult to calibrate, and the pages of text have to be mounted, one by one, in a very precise fashion. The approach described in this paper is much easier and more precise.

Computers also have been used to present successive words of a sentence one (or more than one) word at a time in serial fashion. These tasks can either be paced by the computer (e.g., Potter, 1984) or by the subject (e.g., Mitchell, 1984). Such approaches are very useful, but it is possible that subjects' strategies vary considerably from those used when large text segments are presented and subjects are free to look at lines in any sequence.

The following methodology circumvents many of the above problems. The student is free to study in a manner similar to that used for courses, and yet detailed information about that manner of study is obtained.

\section{EQUIPMENT REQUIREMENTS}

The system includes an IBM Personal Computer with 64K RAM, two 360K disk drives, an AST Megaplus board with 192K RAM and a real time clock, an IBM Color/Graphics Monitor Adapter board (which has the light pen port), a Symtec ${ }^{1}$ Touch-Ring light pen, and a Zenith Model ZVM123 green screen monitor. The pen serves four functions: to indicate which line of text the subject is currently examining, to change a line of text to reverse video (referred to here as highlighting), to change from one page of text to another, and to select the answer for multiple choice questions.

The Symtec pen comes equipped with a ring near its tip which, when touched, enables subjects to highlight and to answer questions. Although unnecessary, we prefer to deactivate the touch ring by inserting a $1 \mathrm{~K}$ resistor between Pins 3 and 4 of the 6-pin connector that connects the light pen to the IBM Color/Graphics Monitor board. A hand-held switch is then inserted between Pins 5 and 6 of the same connector. These modifications permit subjects to operate the switch with one hand and the pen with the other.

To run the program, the system must have at least $128 \mathrm{~K}$ RAM, two disk drives ( $160 \mathrm{~K}$ is less convenient but sufficient), a light pen port, and a real time clock. Since IBM does not produce a real time clock, an after market board is required. The programs described here are written for an AST Megaplus board, but they will work on other AST boards (except the MPII and MonographPlus).

The Symtec pen works with any monitor that does not have the slow P39 phosphors. Best operation is obtained with color, black and white, or green screen monitors; we use the Zenith ZVM123. Although not strictly required, it is desirable to use a stand to hold the monitor in a nearly horizontal position. The stand puts the text in a position similar to that used in normal reading, increases the subject's accuracy in keeping the pen pointed to the correct line, and reduces the fatigue that results from holding a hand up for long periods of time. The stand we use is shown in Figure 1.

\section{COMPUTER PROGRAMS}

The three required programs are written in BASICA and compiled for faster execution. Each program is menu driven and easy to use by inexperienced operators. The programs accommodate passages to 98 pages in length and any number of test questions within the limits of memory and disk capacity. 


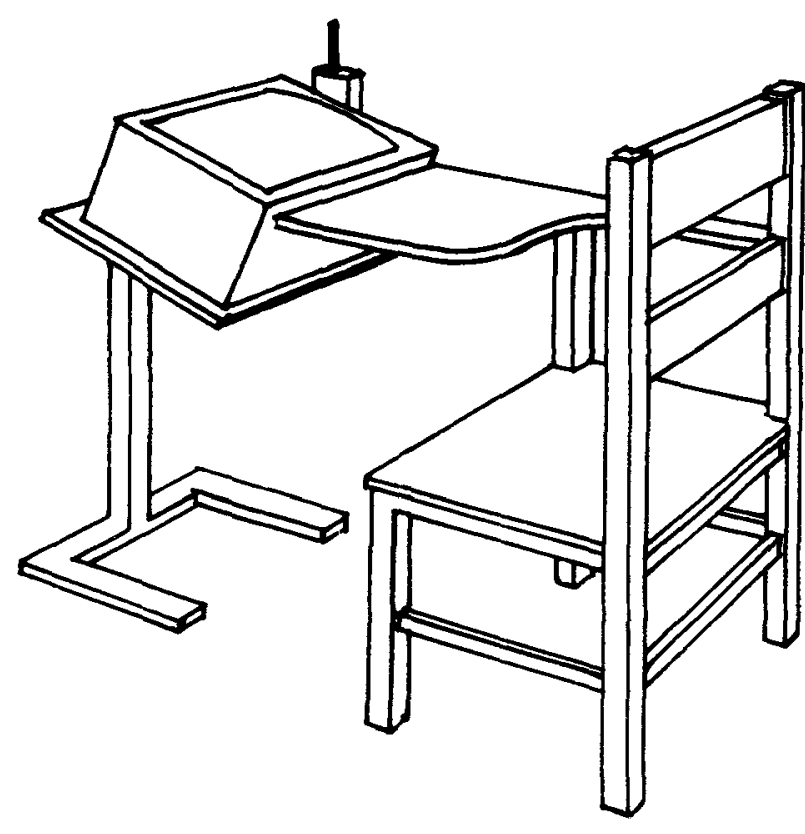

Figure 1. View showing the stand used to hold the monitor. A large plywood panel is normally situated to the right of the chair and stand. The panel provides a visual separation of the experimenter and subject.

\section{Study Program}

One program presents the text and saves the time and frequency data for study sessions. Program execution begins with a series of questions pertaining to the filename of the passage, the subject number (from one to seven digits and/or letters in any combination), whether highlighting will be used ( $Y$ or $N$ ), whether it is the first session for the subject ( $\mathrm{Y}$ or $\mathrm{N}$ ), and whether it is a review session ( $\mathrm{Y}$ or $\mathrm{N}$ ). Then a blank screen appears and all is ready for the experimental session.

When a subject is ready, pressing any key presents the first page of text, a sample of which is shown in Figure 2 . There is a "light band" on both sides of the text. This band of light is three characters wide and extends from the top of the page to the bottom. The purpose of the band is to provide a solid field of light to increase the ease of using the light pen.

The subject is instructed to keep the light pen on the light band beside the line of text being studied. An arrow, shown in Figure 2 at the beginning of the first line of the second paragraph, indicates the line upon which the pen is positioned. Once the pen is beside one line, time on that line starts accumulating until the pen is moved to a different line. Time is recorded to $.01 \mathrm{sec}$, but the accuracy is probably more on the order of .1 sec because of slight delays in reading the clock when the program is performing other activities.

A trap eliminates times too brief to represent actual study. The trap can be set at any value; we used $.3 \mathrm{sec}$, assuming that this represents the minimum time for an eye fixation; any time less than $.3 \mathrm{sec}$ on a line is ignored.
If a subject wants to highlight a line by changing it to reverse video, the switch is pushed while the pen is beside that line. Previously highlighted lines can be changed back to normal video by the same method. Either the left or right light band may be used depending on the handedness of the subject. The program saves any highlighting for later use, such as in a review session.

Subjects can study the lines in whatever order they wish. There is an auditory "beep" when the pen encounters a new line, and an intermittent buzz to warn subjects and the experimenter if the pen moves off the band.

The bottom line of the screen permits changing pages by placing the pen on the square of light beside the appropriate symbol and pushing the light pen switch. A down arrow displays the next page and an up arrow the previous page. In addition, the subject can skip to any page in the passage by entering the appropriate number. For example, Page 23 can be displayed by pushing the switch first when the pen is on 2 , then on 3 , and finally on $E$ (for execute). The left arrow deletes the previous entry. The screen is blank while the chosen page is being loaded into a buffer, and then the entire page appears at once on the screen. There is a blank interval of about $4 \mathrm{sec}$ from the time the page has been selected until it appears on the screen.

When a study session is finished, the subject selects 99 and $E$ from the bottom of the page. A prompt asks if the results are to be saved. The response of $Y$ saves the results, including times, frequencies, and the lines that were highlighted. Informal questioning and observation reveal that subjects readily learn to follow all instructions, and find the use of the pen unobtrusive and enjoyable.

\section{Test Program}

A second program presents the test questions and saves the answers on disk. The program accommodates any number of questions within the memory limitations of the computer and disk. Multiple-choice questions are displayed on the screen with a square of light beside each possible response. The subject selects the answer by placing the pen on the light square beside the chosen answer and activating the light pen switch. Display parameters that can be set by the experimenter are the reading time during which the switch cannot be activated (from 0 to any number of seconds), the time of a beep warning that only $5 \mathrm{sec}$ remain, and the total time from the beginning of one question to the beginning of the next. We use a 15 -sec period before the switch is activated, 10 more seconds before the warning beep, and $30 \mathrm{sec}$ overall. As soon as the subject selects an answer, the question disappears; the screen is blank until the next question appears. This process continues until all questions have been presented, at which point the results are automatically saved.

The program also provides for use of fill-in-the-blank questions. If fill-ins are used, the subject's responses must be recorded by hand, scored, and then entered into the computer by picking the appropriate choice on the menu and following the instructions. 


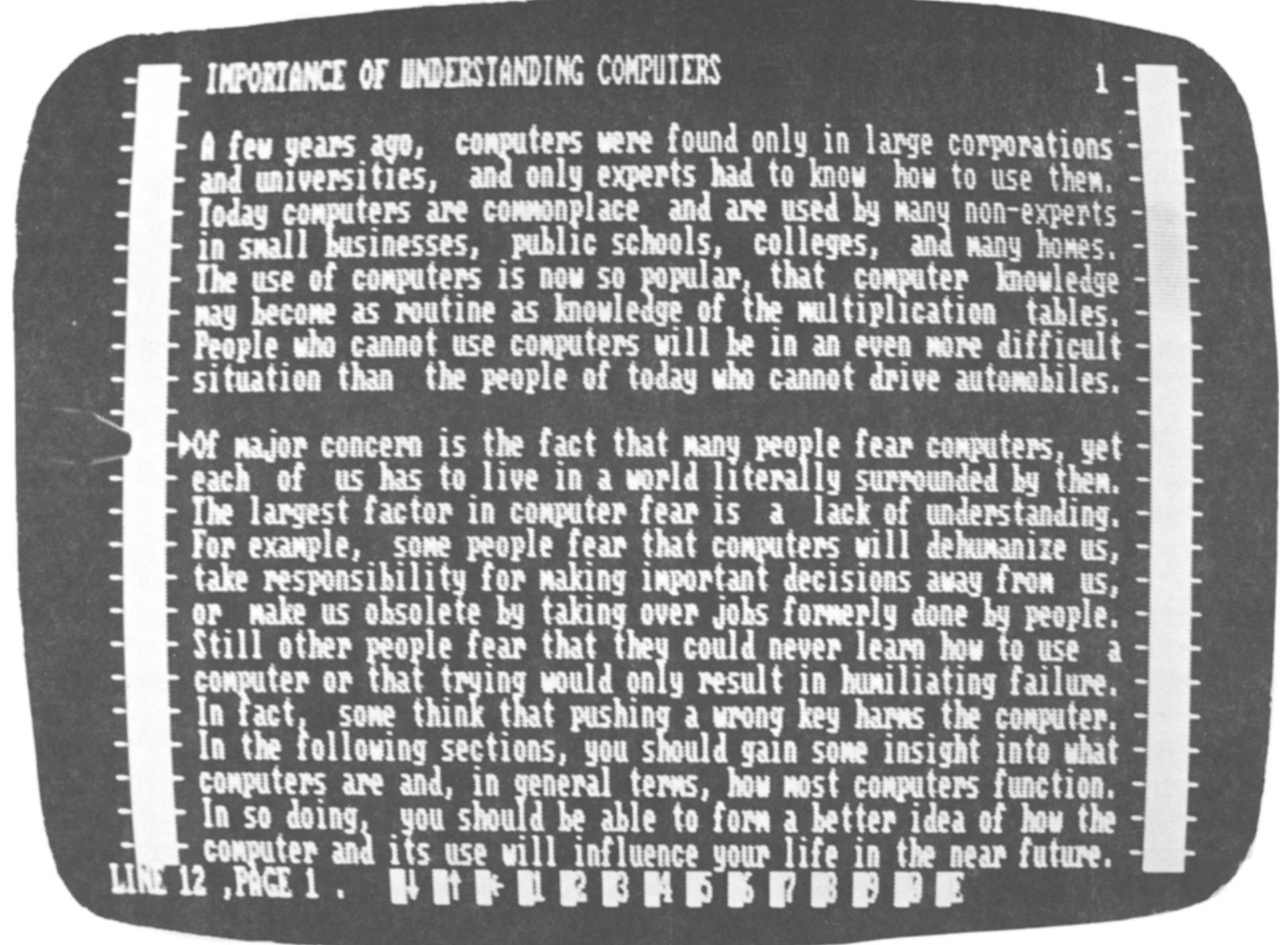

Figure 2. A sample page of text as it appears to the subject on the monitor. The arrow left of the first line of the second paragraph indicates that the light pen is on this line. A status line in the lower left corner indicates the number of the line corresponding to the pen position.

\section{Calculations and Printout}

A third program permits the user to specify headings, important lines (based on whatever criteria the experimenter wants), supporting lines, lines having questions, blank lines (lines separating paragraphs), number of pages in text, and number of questions. This program also performs the calculations and makes the printout of the results.

Raw data printout. The printout begins with a lineby-line listing of the line number in the text followed by an $\mathrm{H}$ if that line was highlighted, the cumulative study time on the line, the number of different times the subject studied that line, the cumulative review time, and the number of times the line was studied during review. One sheet of printout summarizes six screen pages of text.

The answers to multiple-choice test questions appear on a separate sheet. Each question number is followed by a letter (a, b, c, or d) indicating the answer picked, and $a+$ if that answer was correct. If a fill-in-the-blank test is used, this page consists of the question number followed by a 1 signifying a correct response or a 0 for an incorrect answer.
Summary statistics printout. The first page of the summary statistics printout for one sample subject is shown in Table 1. As can be seen, the passage is divided into headings, blanks, and text. Also presented are all possible combinations of important vs. supporting lines, lines with questions vs. those without questions, lines highlighted vs. those not highlighted, and lines from which correctly answered questions were derived vs. those from which incorrectly answered questions were derived.

Two statistics in the middle of the table require clarification. The standard deviation of times on text lines can be used to determine whether a subject emphasizes some lines more than others (producing a large standard deviation), or whether all lines are emphasized almost equally (producing a small standard deviation). Quality of study time is the percentage of the total study time on all lines that was spent on important lines. The greater this percentage, the higher the study quality.

The lower portion of Table 1 presents the number of lines of each type that were in the text (first column), followed by the frequency with which a subject studied, looked back to, and reviewed each type of line. The third 
column contains what have been termed "lookbacks", (Alessi et al., 1979). Basically, the values in this column represent the number of lines of each type to which subjects looked back. Lookbacks are based on, but different from, the values of $\mathrm{N}$ shown in the raw-data printout. The number of lookbacks are derived by subtracting 1 from the $\mathbf{N}$ values in the raw data. The $\mathbf{N}$ values have the advantage of distinguishing between lines that were studied once and those not studied at all. The table makes it easy to compare the number of lines across categories.

Table 2 shows the second page of the summary statistics printout from the sample subject. The blanks on the left are to be read by substituting the column headings into the blanks. Thus, the top value in the middle column

Table 1

Page 1 of the Summary Statistics Printout: Time and Frequency as Functions of Line Type

\begin{tabular}{|c|c|c|c|c|}
\hline \multirow[b]{2}{*}{ Type of Line } & \multicolumn{2}{|c|}{ Study Time } & \multicolumn{2}{|c|}{ Review Time } \\
\hline & Sum & Mean & Sum & Mean \\
\hline All lines & 3171.47 & 4.72 & 364.44 & 0.54 \\
\hline Heads & 1.82 & 0.07 & 22.72 & 0.81 \\
\hline Blanks & 31.03 & 0.44 & 18.14 & 0.26 \\
\hline Text & 3138.62 & 5.48 & 323.58 & 0.56 \\
\hline Supporting & 2150.04 & 5.05 & 218.29 & 0.51 \\
\hline Highlighted & 490.88 & 6.63 & 87.66 & 1.18 \\
\hline Nonhighlighted & 1659.16 & 4.71 & 130.63 & 0.37 \\
\hline Important & 988.58 & 6.73 & 105.29 & 0.72 \\
\hline Without questions & 671.24 & 6.92 & 65.35 & 0.67 \\
\hline Highlighted & 396.07 & 8.25 & 47.02 & 0.98 \\
\hline Nonhighlighted & 275.17 & 5.62 & 18.33 & 0.37 \\
\hline With question & 317.34 & 6.35 & 39.94 & 0.80 \\
\hline Correct & 180.94 & 7.87 & 14.04 & 0.61 \\
\hline Highlighted & 120.34 & 13.37 & 4.92 & 0.55 \\
\hline Nonhighlighted & 60.60 & 4.33 & 9.12 & 0.65 \\
\hline Incorrect & 136.40 & 5.05 & 25.90 & 0.96 \\
\hline Highlighted & 44.46 & 4.94 & 9.40 & 1.04 \\
\hline Nonhighlighted & 91.94 & 5.11 & 16.50 & 0.92 \\
\hline \multirow{4}{*}{\multicolumn{2}{|c|}{$\begin{array}{l}\text { Mean time on all highlighted lines } \\
\text { Mean time on all nonhighlighted lines } \\
\text { Standard deviation of times on text lines } \\
\text { Quality of study time }\end{array}$}} & \multirow{4}{*}{\multicolumn{2}{|c|}{$\begin{array}{r}7.5120 \\
4.8200 \\
3.4698 \\
31.171 \%\end{array}$}} & 1.06 \\
\hline & & & & 0.4030 \\
\hline & & & & 0.9975 \\
\hline & & & & \\
\hline
\end{tabular}

Quality of study time

\begin{tabular}{crrrr} 
& \multicolumn{4}{c}{ Number of Lines } \\
\cline { 2 - 5 } & & & Looked \\
& In Text & Studied & Back to & Reviewed \\
\hline All lines & 672 & 602 & 112 & 274 \\
Heads & 28 & 4 & 0 & 14 \\
Blanks & 71 & 32 & 1 & 19 \\
Text & 573 & 566 & 111 & 241 \\
Supporting & 426 & 419 & 73 & 162 \\
Highlighted & 74 & 74 & 26 & 48 \\
Nonhighlighted & 352 & 345 & 47 & 114 \\
Important & 147 & 147 & 38 & 79 \\
Without questions & 97 & 97 & 28 & 52 \\
Highlighted & 48 & 48 & 15 & 32 \\
Nonhighlighted & 49 & 49 & 13 & 20 \\
With questions & 50 & 50 & 10 & 27 \\
Correct & 23 & 23 & 7 & 10 \\
Highlighted & 9 & 9 & 5 & 4 \\
Nonhighighted & 14 & 14 & 2 & 6 \\
Incorrect & 27 & 27 & 3 & 17 \\
Highlighted & 9 & 9 & 2 & 8 \\
Nonhighlighted & 18 & 18 & 1 & 9 \\
\hline
\end{tabular}

Table 2

Page 2 of Summary Statistics Printout: Summary of Frequency Data and Test Results

\begin{tabular}{lccc}
\hline & \multicolumn{3}{c}{ Type of Response } \\
\cline { 2 - 4 } & $\begin{array}{c}\text { High- } \\
\text { lighted }\end{array}$ & $\begin{array}{c}\text { Looked } \\
\text { Back to }\end{array}$ & Reviewed \\
\hline \% of important lines that were - & 44.90 & 25.85 & 53.74 \\
\% of supporting lines that were - & 17.37 & 17.14 & 38.03 \\
\% of highlighted lines that were & & 34.29 & 65.71 \\
\% of _ lines that were important & 47.14 & 33.93 & 28.83 \\
\% of _ lines that were supporting & 52.86 & 65.18 & 59.12 \\
\% of __ lines having questions & 12.86 & 8.93 & 9.85
\end{tabular}

(Read above lines by substituting column headings, one by one, into the blanks.)

Total number of highlighted lines

Total number of nonhighlighted lines

Quality of highlighting

Total number correct

Number highlighted and correct

Number nonhighlighted and correct

$\begin{array}{cc}140 & \\ 433 & \\ 30.084 & \\ 14 & 50.00 \% \\ 6 & 42.86 \% \\ 8 & 57.14 \%\end{array}$

Probability of a correct answer given

(a) Highlighting of lines questions was based on $\quad 0.5455$

(b) No highlighting of lines questions was based on $\quad 0.4706$

(c) Review of lines the question was based on $\quad 0.4444$

(d) No review of the lines the question was based on $\quad 0.6000$

(25.85) represents the percentage of important lines to which subjects looked back. The value of 12.86 in the bottom of the first column represents the percentage of highlighted lines having questions based on them, and so forth.

At the bottom of Table 2, the total number of highlighted and nonhighlighted lines refers to the study session. Quality of highlighting refers to the observed frequency of highlighting important lines minus the chance frequency, as defined by the following formula:

$$
\mathrm{Q}=\mathrm{NIHL}-\frac{\mathrm{NHL}}{\mathrm{NTL}}(\mathrm{NIL})
$$

where $\mathrm{Q}=$ quality; NIHL = number of important lines that were highlighted; NHL $=$ total number of highlighted lines in the passage; NTL = number of text lines in the passage (excluding heads and blank lines); NIL = number of important lines in the entire passage.

Test data. Compilations of the major test data are shown in the bottom section of Table 2 . The values are self explanatory.

\section{Sample Passage}

Any passage up to 98 pages in length can be used. However, we wrote and will describe a sample passage we find useful. The passage is on the subject of computers, which we judged would be of sufficient interest and usefulness to encourage students to return for multiple-session experiments. The passage meets the following criteria: (A) Each page fills a 24-line computer screen; (B) each page begins with a heading and consists of either two or 
three paragraphs; (C) each line of text is $62-65$ characters long, including spaces between words. If needed, a maximum of three additional spaces per line were added to right justify each line to make the text appear more like printed material; (D) each sentence was 1, 2, or 3 lines long and ended at the right margin, enabling an examination of the time spent on each line of text without the ambiguities that arise if some lines consist of parts of two different sentences; (E) each screen page of text had from 4-6 lines that were judged important. Not one of the many subjects we asked was aware that the text had these features.

Three judges (the first three experimenters) evaluated the text for important lines. The instructions were to select exactly six lines of text per page that best answered three questions that could be asked about the page heading. These questions were as follows: (A) What is - ? (B) What are the main characteristics of _ ? (C) What are the main uses of _ ? The key word(s) of each heading was (were) substituted into the blanks. For each heading, only those questions that made sense for that heading were considered. For example, it makes no sense to ask "What are the main uses of the major divisions of a computer," and so the question would not be used.

Text lines were considered important only if selected by all three judges. If three judges picked four or more lines in common, the page was acceptable. Otherwise, the page was rewritten to place more emphasis on some lines and less on others. The page was then reevaluated by the judges. The end result was 28 screen pages. A sample of two pages is presented in the Appendix. Of course, a different procedure can be used to define important lines or to create an entirely new text if desired.

A detailed structural analysis of a lengthy text is extremely complicated; however, each sentence of the passage was divided into propositions following the procedure described by Thorndyke (1977). Basically, a proposition was defined to be a clause or sentence that had an action or stative verb. Supporting and important lines were similar. The percentages of supporting sentences that were 1,2 , or 3 lines long were 44,51 , and 5 ; the corresponding values for important sentences were $45 \%, 52 \%$, and $3 \%$. The mean number of propositions for supporting (important) lines was $1.81(1.78)$ for 1 -line sentences, $1.67(1.69)$ for 2-line sentences, and $1.72(1.33)$ for 3line sentences. Because there were very few 3 -line sentences (only 3 were important sentences), there is more variability for these scores. Based on all sentence lengths, the mean number of propositions per line was 1.71 for supporting lines and 1.68 for important lines.

The division into important and supporting lines is given empirical support by data collected from 36 freshmen who studied the sample passage for two 30-min sessions separated by a 5 -min break. The subjects were brought back two days later for a 10-min review and given the multiplechoice test described below. Subjects spent longer per important line than per supporting line in original study $(6.00$ sec vs. $4.99 \mathrm{sec})$ and in review $(.93 \mathrm{sec}$ vs. $.62 \mathrm{sec})$. As would be expected, the same amount of time was spent on important lines whether they had questions $(6.01 \mathrm{sec})$ or not $(5.97 \mathrm{sec})$.

A second study using 35 introductory psychology students had two study sessions but no review. Subjects were instructed to highlight from 4-6 lines per page that were important in answering the three questions used by the judges to evaluate importance. The mean times for important and supporting lines were $7.21 \mathrm{sec}$ and $4.52 \mathrm{sec}$, respectively. Moreover, subjects highlighted $59 \%$ of the important lines but only $12 \%$ of the supporting lines. Considerably more time was spent on highlighted lines $(9.19$ sec) than nonhighlighted (4.01).

\section{Test Over the Sample Passage}

The mean test score was 17.06 for students taking the immediate test vs. 14.70 for subjects who had the test after a two-day retention interval. Thus, neither floor nor ceiling effects are present.

The test consists of multiple-choice test questions written following the guidelines of Anderson (1972). Anderson called these paraphrase or transformed paraphrase questions. All substantive words (nouns, verbs, and modifiers) were changed except the technical terms (e.g., printer, pixel), and a few less technical terms (e.g., key on a keyboard). A transformed paraphrase question involves a reordering of paraphrases. Examples of the questions are shown in the Appendix.

Each question represented a paraphrasing of an entire sentence (or, rarely, nearly all of a sentence) from the original text, with the omission of one word or short phrase indicated by an underlined blank. The four choices for each multiple-choice question were approximately equal in length. The choices were randomized for each question with the restriction that, within each block of four, the correct answer would appear once as a, b, c, and d. Alternatives such as "all of the above" and "none of the above" were avoided.

An item analysis of the questions was obtained by having 37 subjects take a pretest in which they were asked to define seven common computer terms, read a 28-page printed version of the passage for a total of $50 \mathrm{~min}$, paying particular attention to the main points covered under each heading, and take a 28 -item test over the passage, with one question from each page of the passage, and $30 \mathrm{sec}$ to answer each item. The questions were in booklets with one question per page.

In most classroom situations, many students have some knowledge of the subject at the beginning of the course. This knowledge showed in our pretest: The mean percentage of correct answers was 33.4.

The difficulty level and discrimination index were determined for each multiple-choice item. The difficulty level ranged from $27 \%$ to $89 \%$ correct, with a mean of $16.32(58.3 \%)$ and a standard deviation of 4.94 . Thus most items avoided floor or ceiling problems, which is important for researchers who plan to examine different training techniques. The discrimination indexes, which 
ranged from -.01 to .65 , were satisfactory. In fact, 20 of the 28 items had values of . 30 or higher and are considered to be good to very good items (Guilford, 1965).

\section{BENEFITS OF THE APPROACH}

The experimental procedures presented in this paper provide the following benefits: (A) Detailed time and frequency data can be collected easily even for very long passages. (B) There is great flexibility in the types of study that can be examined, including reading, review, lookbacks, metacognition, and test performance, to name just a few. The approach is especially suited to testing the selective attention hypothesis. (C) Passages can be presented in a "normal" fashion in that subjects are free to go to any line on any page at any time. (D) The task is relatively unobtrusive and enjoyable for the subject. The use of the light pen is similar to pencils or markers and hence similar to what many subjects use when studying. (E) A passage and test are provided for researchers who do not wish to generate their own. (F) Times, frequencies, and many other statistics are determined automatically and objectively to facilitate data collection and comparisons among different laboratories.

\section{AVAILABILITY}

The programs and documentation can be obtained on disk by sending $\$ 15.00$, to cover the cost of disks and mailing, to the first author. Checks should be made payable to Washington State University Department of Psychology. Unless specified otherwise, it is assumed that the requestor has double sided, double density disk drives. The sample text and test questions are included.

\section{REFERENCES}

Alessi, S. M., Anderson, T. H., \& Goetz, E. T. (1979). An investigation of lookbacks during studying. Discourse Processes, 7, 197-212.

ANDERSON, R. C. (1972). How to construct achievement tests to assess comprehension. Review of Educational Research, 42, 145-170.

Anderson, R. C., Pearson, P. D. (1984). A schema-theoretic view of basic processes in reading. In P. D. Pearson (Ed.), Handbook of Reading Research (pp. 255-292). New York: Longman.

Anderson, T. H., \& Armgruster, B. B. (1984). Studying. In P. D. Pearson (Ed.), Handbook of Reading Research (pp. 657-680). New York: Longman.

ANNIS, L., \& DAvis, J. K. (1978). Study techniques and cognitive style: Their effect on recall and recognition. Journal of Educational Research, 71, 175-178.
Crouse, J. H., \& Idstein, P. (1972). Effects of encoding cues on prose learning. Journal of Educational Psychology, 63, 309-313.

Britton, B., Meyer, B., Simpson, R., Holdredge, T., \& Curry, C. (1979). Effects of the organization of text on memory: Tests of two implications of a selective attention hypothesis. Joumal of Experimental Psychology: Human Learning and Memory, 5, 496-506.

Brown, A. L., \& SMILEY, S. S. (1978). The development of strategies for studying texts. Child Development, 49, 1076-1088.

Fass, W., \& Schumacher, G. M. (1978). Effects of motivation, subject activity, and readability on the retention of prose materials. Joumal of Educational Psychology, 70, 803-807.

FAW, H. W., \& WALLER, T. G. (1976). Mathemagenic behaviours and efficiency in learning from prose materials: Review, critique and recommendations. Review of Educational Research, 46, 691-720.

Garner, R., MACrEAdy, G. B., \& Wagoner, S. (1984). Readers' acquisition of the components of the text-lookback strategy. Journal of Educational Psychology, 76, 300-309.

GaRNER, R., \& REIS, R. (1981). Monitoring and resolving comprehension obstacles: An investigation of spontaneous text lookbacks among upper-grade good and poor comprehenders. Reading Research Quarterly, 16, 569-582.

GUILForo, J. P. (1965). Fundamental Statistics in Psychology and Education. New York: McGraw-Hill.

MitchelL, D. C. (1984). An evaluation of subject-paced reading tasks and other methods for investigating immediate processes in reading. In D. E. Kieras \& M. A. Just (Eds.), New Methods in Reading Comprehension Research (pp. 69-89). Hillsdale, NJ: Erlbaum.

PiCherT, J. W., \& ANDERSON, R. C. (1977). Taking different perspectives on a story. Journal of Educational Psychology, 69, 309-315.

PotTer, M. C. (1984). Rapid serial visual presentation (RSVP): A method for studying language processing. In D. E. Kieras \& M. A. Just (Eds.), New Methods in Reading Comprehension Research (pp. 91-118). Hillsdale, NJ: Erlbaum.

Raphael, T. E., Myers, A. C., Tirre, W. C., Fritz, M., \& FreeBODY, P. (1981). The effects of some known sources of reading difficulty on metacomprehension and comprehension. Joumal of Reading Behavior, 13, 325-334.

REynolds, R. E., StANDiford, S. N., \& ANDerson, R. C. (1979). Distribution of reading time when questions are asked about a restricted category of text information. Journal of Educational Psychology, 71, 183-190.

Sulin, R. A., \& Dooling, D. J. (1974). Intrusion of a thematic idea in retention of prose. Journal of Experimental Psychology, 103, 255-262.

THORNDYKE, P. W. (1977). Cognitive structures in comprehension and memory of narrative discourse. Cognitive Psychology, 9, 77-110.

Wollen, K. A., Cone, R. S., Britcher, J. C., \& Mindemann, K. M. (1985). The effect of instructional sets upon the apportionment of study time to individual lines of text. Human Learning: Journal of Practical Research \& Applications, 4, 89-103.

\section{NOTE}

1. Symtec, 15933 West 8 Mile, Detroit, MI 48235. 
APPENDIX

Two Pages and Questions from the Sample Passage

\begin{tabular}{ll}
\hline 385 & DISK FORMATTING \\
386 & 9
\end{tabular}

BASIC will be used to illustrate the fundamentals of programming. We will consider the notion, important in every language, that a computer must execute instructions in a particular sequence. In BASIC, the execution of statements in the correct sequence is made possible by giving every statement its own unique number. The CPU first locates and executes the statement with the lowest number, then the statement having the next lowest number, etc. Any numbers can be used provided they contain no decimal places. However, it is customary to give each statement a number that is divisible by 10 and is ten larger than the previous line number. Thus, a typical sequence might be $120,130,140,150,160$, etc Separating statements by 10 enables a programmer to insert new instructions between statements if the need is discovered later.

Another fundamental notion is substituting one value for another. There are many situations in which such exchanges must be made. For example, consider the following BASIC statement: $80 \quad N=N+1$, where $N$ stands for some number that can have different values. This statement tells a computer to take the old value of $N$ (which is the $\mathrm{N}$ to the right of the equals sign), to add 1 to it, and to call the result the new value of $N$ (the $N$ left of the = sign). In other words, the new value of $N$ would be the old value plus 1 .

17. The basic procedure of setting up a disk, in order to be able to store data on it, is usually called _.
a. booting up
b. organizing
c. formatting
d. flowcharting

22. The instruction that the microprocessor finds and carries out first is the one having -

a. a line number of 10

b. the smallest line number

c. the largest line number

d. a START command

Note: The line numbers are not presented to the subject. Boldfaced line numbers refer to lines designated as important, and a " $Q$ " after the line indicates that a question was based on that line.

(Manuscript received November 14, 1984;

revision accepted for publication May 1, 1985.) 